\title{
From Confocal Microscope to Virtual Reality and Computer Games; technical and educational considerations
}

Craig J Daly. School of Life Sciences, College of Medical Veterinary \& Life Sciences, University of Glasgow, Glasgow GI28QQ

\section{Abstract}

Will it ever be possible to visualise a microscopemounted live cell in virtual reality in real time? It is possible, and we may be closer than many think. The article outlines the workflow involved in taking confocal laser scanning microscopy (CLSM) data through to fully immersive virtual reality (VR) and interactive games. For the past 30 years, the size of standard CLSM data sets has generally exceeded the capabilities of standard desktop PCs and thus hindered the ability to process them in creative ways. Modern gaming PCs, high end graphics cards and VR headsets have changed all that. A moderately priced desktop PC can now extend the value of a CLSM data set through the use of $3 \mathrm{D}$ animation software and game engines. There are considerable technica processes that CLSM datasets need to pass through as there is no single piece of software that will cover the entire sequence. However, most of the
\end{abstract}

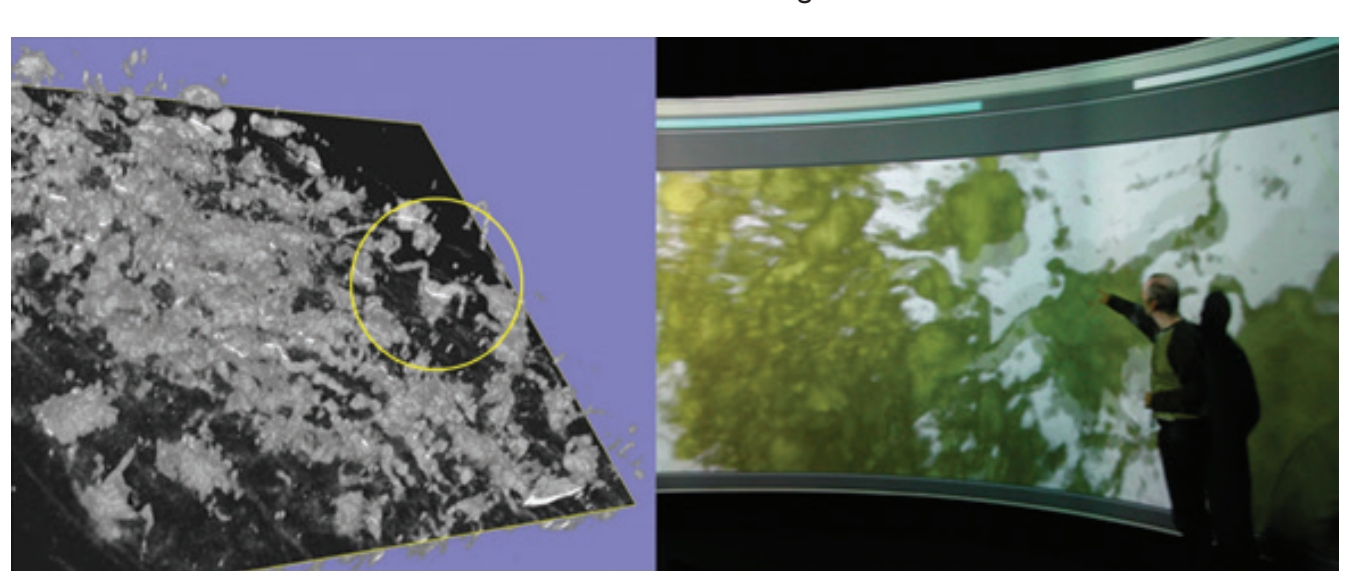

Figure I. A confocal-derived volumetric rendering (from IMARIS software) of the adventitial surface of an artery and a peri-vascular nerve (circled, left). The author (right) in 2003 presenting the same data set in aVR suite that used stereo projection and $3 \mathrm{D}$ glasses
screen to produce $a 3 \mathrm{D}$ view of the data. The stereo (blurry) screen view was switched off for the purposes of photography. full box of 10 floppy disks. The PC used to drive the CLSM struggled to do much more than create a maximum intensity projection of the data and rotate it around the $x$ or $y$ axis. To achieve anything close to $3 \mathrm{D}$ rendering required the purchase of some fairly expensive software and hardware (IMARIS $\mathrm{vI}$, VoxelView, FiRender and a Silicon Graphics Onyx workstation). Visualisation in 3D could cost around $50 \%$ of the cost of the CLSM itself. Translate that into today's costs for a standard CLSM and you have an idea of the scale of the problem. From about 1993-2002 computing power increased slowly but CLSM systems were producing more channels of data at ever increasing speeds and image resolution. So, file sizes continued to get larger.

In 2003 we were lucky enough to gain access to the Glasgow Science Centre Virtual Reality suite (figure I). This was partly due to having a collection of 3D data sets that were in a suitable format. The resulting $3 \mathrm{D}$ (stereo projection) experience was less than impressive; but we felt like pioneers. Particularly since this facility was powered by something called an SGI Onyx Reality Monster, which cost about the same as a luxury penthouse.

In the following 10 years, desktop computing would catch up with the capability of CLSM systems. This was largely due to the popularity of PC gaming, lowering costs of RAM and the availability of extremely powerful graphics cards. On the software side, the growing popularity of digital 3D art, animation and modelling also provided tools that could be of significant use to CLSM users (Daly et al., 2014). The purpose of the present article is to introduce a selection of software tools and techniques that are routinely used by $3 \mathrm{D}$ animators and game designers but may not be obviously relevant to microscopists. The general aim is to encourage CLSM users, who archive vast quantities of 3D data, to think about how that information could be more fully exploited.

Thresholding \& Segmentation

$3 \mathrm{D}$ animation and game engine software require data that is in the form of a wire mesh. Processing and manipulation of the mesh is described in the sections that follow. The first stage in creating a mesh (or so-Surface) is thresholding and segmentation (T\&S). The process of T\&S requires the identification of specific structures within a volume. For CLSM data this will generally be based on intensity or colour of an object. There are many techniques for achieving T\&S, some of which can be quite elaborate in their attempts to split touching objects (Luo et al. 1998) or to identify pathological regions within medical images (Malood et al., 2018). Most microscopy and image analysis users will be very familiar with these techniques and so this section will be brief and touch on a few utilities that the author has found useful.

IMARIS image analysis software (Bitplane) is a very powerful visualisation and analysis tool which has a simple user interface and a good automated T\&S routine. At the time of writing the current version is 9.2.1, which was used for evaluation. The automatic T\&S function works on-the-fly and is particularly good for datasets with strong contrast between objects. Once an iso-surface is created it can be exported in .wrl or .iv formats. The .wrl format can be read by MeshLab (see below)

AMIRA image analysis software (Thermo Scientific) is also a very advanced and powerful 3D visualisation and analysis package. At the time of writing the current version is 6.7, which was used for evaluation. The T\&S module offers some very useful tools that include paint brushes, lasso, magic wand, contour, blow, top-hat, watershed and histogram for intensity (threshold) selections. These are particularly useful for 'difficult to segment' datasets. At the iso-surface generation stage,AMIRA also reports the dimensions (size) of the geometry and offers a mesh-reduction option. It is particularly useful to have this reduction facility within the AMIRA package as it can, in some cases, mean that one step can be missed prior to exporting to animation or games software.

There are a few freely available packages that can be extremely helpful. The most common is Image//Fiji which most, if not all, microscopists will be aware of The 3D plugins (3D viewer and Volume Viewer) are 
useful for visualisation but limited in their 3D T\&S capabilities.

of the packages mentioned above with representative data sets of the type most likely to be used.

\section{Working with Meshes}

is a particularly good package for name suggests it provides a means of segmenting $3 \mathrm{D}$ volume to create a surface. The surface can then be exported in a variety of formats (.vtk, .fac., .pts. .val and .stl).

3DSlicer is very powerful and free. This 3D medical imaging package is currently at v4.10 at the time of writing and whilst mainly focused on MR \& CT image data, it can be used successfully with microscope-based TIFF and PNG series. 3DSlice has been continually developed over 20 years an now offers an impressive set of T\&S tools alongside a growing suite of plugins which now includes VR module. Although not extensively used by the author, it is anticipated that 3DSlicer would be more than capable of inserting into the workflow detailed in this article (Figure 2).

T\&S are essential components of the workflow towards fully immersive interaction in virtual reality. Therefore, it is worth spending time evaluating each

\section{Image Capture}
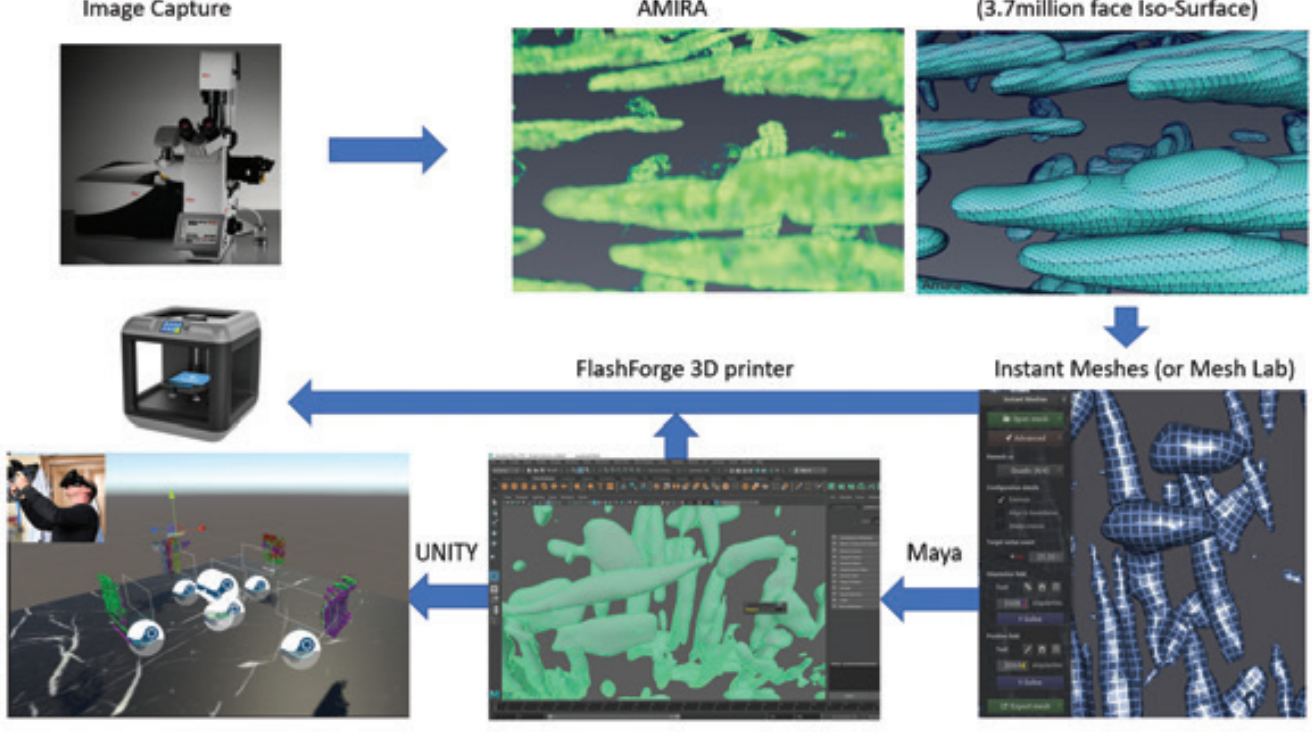

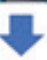

The overall success of the workflow described here rests largely on the quality of the iso-surfaces (meshes). The goal is always to produce a mesh which comprises the minimum amount of geometry and stil accurately represents the original objects. In some cases, the surface mesh from a large single channel data set from a modern confocal to facilitate manipulation by other software ass geometry reduction using AMIRA' and possible conversion to quads. Instant Meshes, Mesh Lab, Meshmixer and other applications provide tools to reduce and quadrate the triangular faces. Animation and modelling software (eg Maya, ZBrush Figure 2. The data workflow from confocal microscope to 3D printing and virtual reality. Data (a multi-channel z-series) is collected via confocal microscopy and passed to the thresholding and segmentation package of choice. In this case it is AMIRA and the dataset is the tunica media
of a mouse mesenteric artery. The objects are smooth muscle cell nuclei (typically 25 um $x 2 u m$ size). Thresholding and segmentation routines create an iso-surface made from vertices and faces (wireframe mesh). The resulting mesh is processed and sent to either a $3 \mathrm{D}$ printer or to

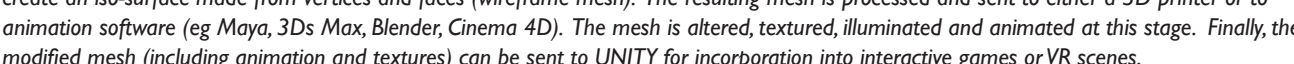
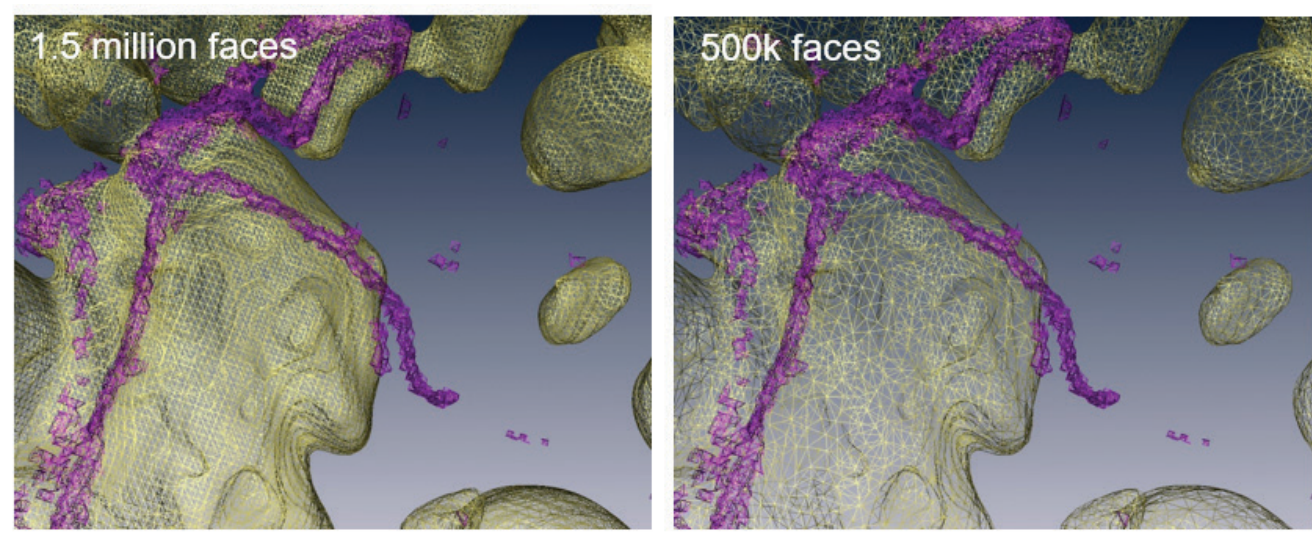
Figure 3. A sympathetic nerve fibre (purple) makes contact with a perivascular adipocyte (yellow). The original iso-surface, generated through
thresholdding and segmentation produced a mesh containing 1.5 million faces. Reducing the poly-count by $33 \%$ (500k faces) results in little, if any, loss of anatomical detal

and Mudbox) can work with triangles or quads but the preference (among 3D artists) appears to be for quads for smoother subdivisions and manipulation. Internet forums are filled with debate on the topic. The choice of working with triangles or quads will depend on the task at hand. The author's preference is for a quad mesh where possible but it is not always essential. It should be noted though that most microscope or imaging-based software (i.e. AMIRA, IMARIS and 3DSlicer) will create a triangulated isosurface mesh by default. This is perfectly acceptable for most applications. Figure 2 shows examples of smooth muscle cell nuclei as triangulated meshes in AMIRA but as quad meshes in both Instant Meshes and MAYA.

Passing through a 'mesh editing' stage offers the opportunity to 'fix' any minor defects. Sometimes a segmentation may not completely capture an entire structure, and this can result in broken edges or holes in a surface. It may be necessary to split two surfaces that are touching, if one will be textured differently or animated separately. Alternatively, two surfaces may need to be joined or smoothed. Often modelling or animation software may complain when a surface contains 'non-manifold' geometry. This could be a vector which has no face or two vectors that are missing a $3^{\text {rd }}$ to complete a face. This can cause problems for some animation or $3 \mathrm{D}$ printing obs. Fortunately, most mesh editors will have tools to address these and similar problems (figure 4). The current version of MeshLab (v20I6.12 at the time of writing, December 2018) offers an exhaustive list of tools for mesh editing and is an essential part of the author's workflow.

\section{D Rendering \& Animation} Volume Rendering

$3 \mathrm{D}$ gaming and design software has radically improved the capability of volume rendering. Previously, microscopists were limited to fairly basic volumetric rendering based on intensity and transparency of voxels. High end Graphics Processing Unit (GPU) cards and modern Computer Generated Imagery (CGI) techniques now offer real-time ray casting to create very realistic looking 3D scenes that can be rendered on-the-fly. Whilst a detailed discussion of ray tracing and ray casting is beyond the scope of this article, the technology seems poised to enter a new

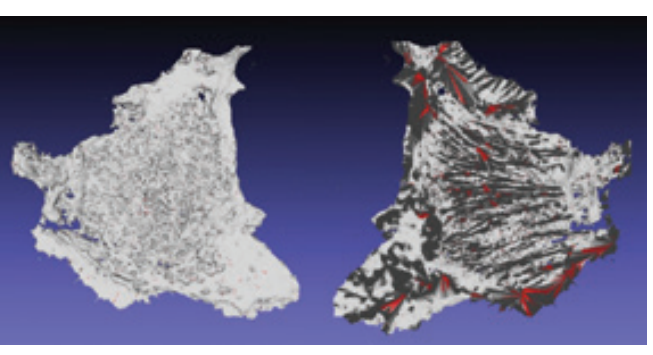

gure 4. Two views of a single COS cell scanned using a Leica SP8 on-manifold geometry. The top surface of the cell (left) has no defects. The underside of the cell (right) displays non-manifold geometry (in red) which requires attention. The cell measures $65.8 \mu \mathrm{m}$ at its longest axis.
Size bars are not shown as the $3 \mathrm{D}$ volumes can have slightly different 


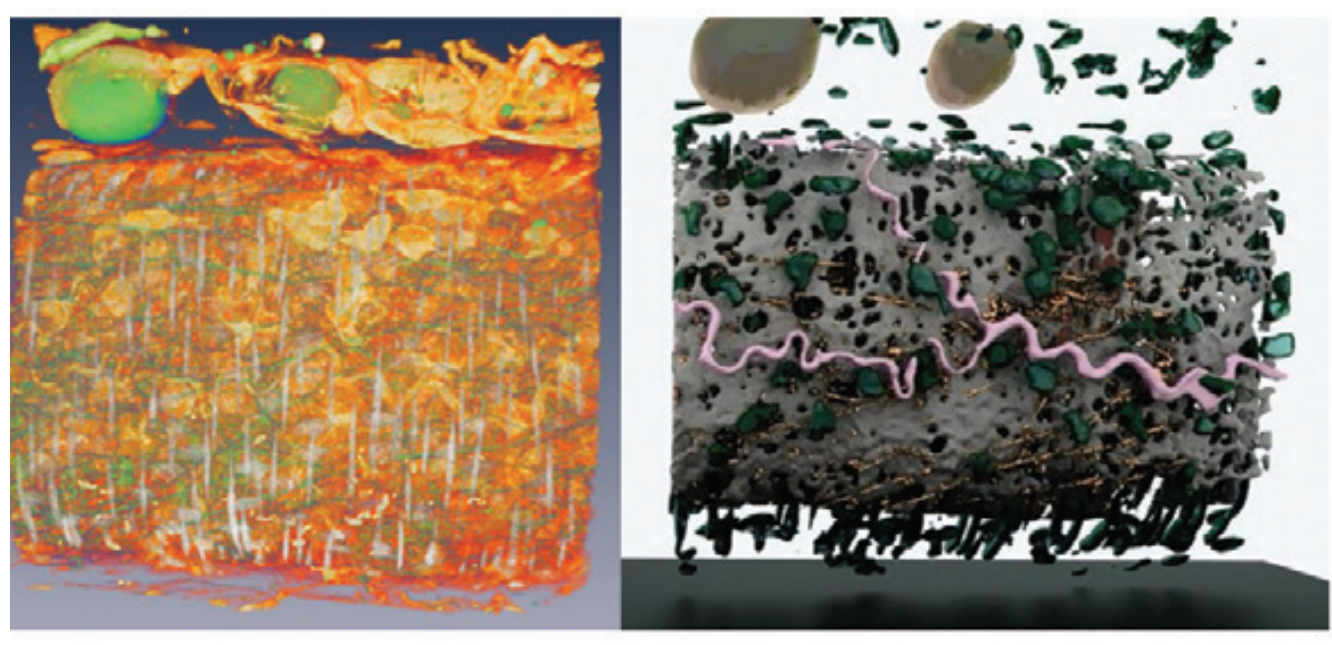

Figure 5. Comparison of voxel rendering with 'Arnold' photorealistic rendering. The image on the left is a 'voltex' rendering of a perfusion-fixed vascular segment (mouse mesenteric artery). It is a 3-channel confocal fluorescence data set comprising cell nuclei (grey, syto 61) alphaladrenoceptor ligand (green, prazosin) and a beta-adrenoceptor ligand (orange, TMR-cgp 2 2177). The volume rendering shows the 3 channels in differing opacities. Right image; the same data set has been rendered using Autodesk Maya 'Arnold' using photorealistic surfaces such as ceramic
(white external elastic lamina, pink autonomic nerve), emeralds (adventitial cell nuclei) and glass (yellow perivascular adipocytes). Size bars are not shown due to the 3D nature of the volumes. For reference, the smooth muscle cell nuclei (grey) in the left image are $\sim 25 u m \times 2 u m$ in size.

phase that will bring a significant leap in both speed and quality of rendering. A top of the range gaming GPU card (eg NVIDIA GeForce RTX 2080) will cost around $t 750$ (December 2018). The author's developments have relied on a cheaper NVIDIA GTX $1070(\sim 6350)$ in a PC with 16Gb of RAM. A recent development in the ophthalmology field has seen one group demonstrate real time immersiveVR visualisation of Optical Coherence Tomography using two GPU cards (NVIDIA GTX 1080 \& 670) for ray casting and additional graphics processing (Draelos et al., 2018). These authors explore the idea of realtime surgery where the surgeon has a VR view of

the live tissue.

\section{Surface Rendering}

Animation software (eg Autodesk Maya) provides 3D rendering options in which real life textures can be used. This approach places a heavy load on the processor as each surface reflection and transparency must be calculated such that shadows fall in the correct place, thus giving materials a realistic look. Maya uses 'Arnold' rendering by default but several other plug-ins are available. A comparison between a standard AMIRA volume rendering and a Maya-Arnold rendering is shown in figure 5 . The Arnold render has the appearance of a real life solid

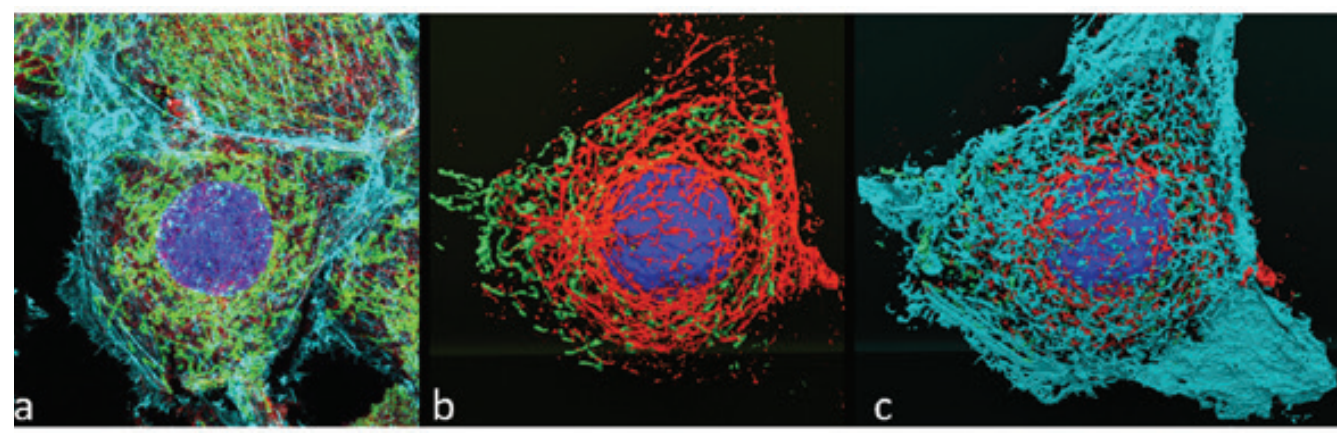

Figure 6. Comparison of Imagej z-projection and Autodesk MAYA 'Arnold' rendering of extracted surfaces. a) 4 channel CLSM (imagef) z-projection of a stained COS cell. Tubulin (cyan), Mitochondria (green), actin (red), nucleus (blue). b) 3D surface model, Arnold rendering of rotated and cropped to remove adjacent cell material. The cell measures $65.8 \mu m$ at its longest axis. Size bars are not shown as the $3 D$ volumes can have slightly different titt angles to facilitate visualisation of specific elements object due to the use of familiar looking materials such as copper wire, ceramic and emeralds. It should be noted that a single view can take several minutes to render, depending on the complexity of light and texture. Therefore a simple 360 degree rotation could take hours to render out as a series of frames that need to then be composited to a movie file.

CLSM users will have their own preference for $3 \mathrm{D}$ processing software. The simplest platform is probably Image//Fiji running 'Volume Viewer' or '3D Viewer'. Both of these ImageJ plugins are very good and offer a range of rendering options. Image] is extremely useful for creating z-projections (figure 6a), altering image depth (16-bit to 8-bit etc) and exporting data in a variety of formats and will therefore easily fit into any workflow.

The limitation of most (if not all) microscope-based $3 \mathrm{D}$ packages is that 'animation' generally refers only to the camera position and altering the visibility of volumes and surfaces. Typically, the user only has control over a single camera that can fly through and around a 3D volume. The output can then be saved as a movie. This is perfectly adequate for presenting the structure of a 3D model. However, what if you want to animate the model itself? You may have a beautiful 3D model of a cell, but you may want to animate its movement, contraction, release or uptake of substance etc. For that you need to convert the data into a form that could be read by dedicated 3D animation software (Daly et al., 2014). Autodesk

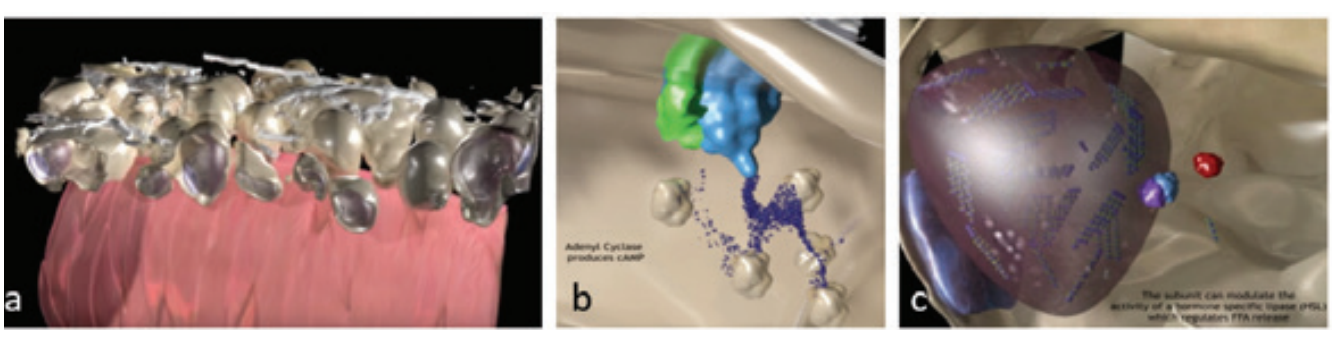
igure 7. Combining confocal microscopy data, 3D protein structures, artistic components and particle flows in a single animation. The figure shows screenshots from a complete animation which can be viewed at the author's You Tube channel. [See www.cardiovascular.org for YouTube nerve supply (white). These structures are captured directly using G ISM The pink smooth muscle cells (tunica media) are drawn and added for context. b) the inner surface of a PVAT cell (confocal data) with adenylate cyclase (blue) and a bound G-protein alpha-subunit (green) activates particle flow of CAMP molecules (purple). The CAMP stream is modified by an invisible wind generator and virtual magnets are placed within the phosphodiesterase molecules (beige) to attract (and degrade) the CAMP. c) The large lipid blob containing the triglycerides has been added to the scene (CLSM-derived framework); the hormone specific lipase (blue and purple) structure was downloaded from the protein data bank (PDB) as 


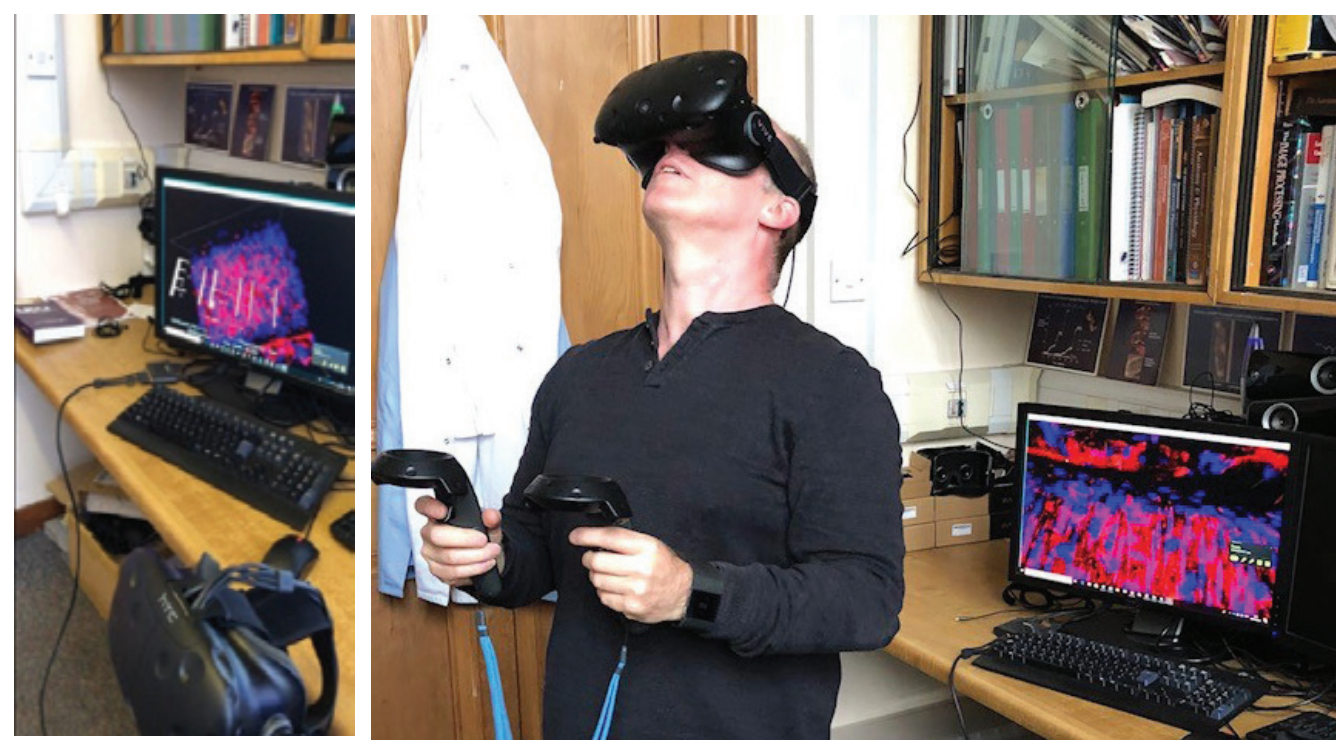

Figure 8.V Visualising volumetric CLSM data in VR using the application 'ConfocallV' ( 1 ww.confocalv.com). The data being visualised by the
author is a confocal volumetric reconstruction of a segment of vascular (arterial) wall. Slider controls shown on the screen on the left image author is a confocal volumetric reconstruction of a segment of vascular (arterial) wall. Slider controls shown on the screen on the left image
enable alterations in brightness, contrast, image depth etc.

The importance of extracting a good wireframe mesh (or surface) has been discussed above. Animation software provides virtually unlimited scope for manipulating and texturing the extracted mesh. I addition, other 'actors' can be added to the scene. These could be in the form of neurotransmitters or cellular vesicles. Essentially, components of the scene that cannot be derived from microscopy or other means instead need to be created (drawn). Figure 7 provides several examples. In figure 7a, the gold coloured cells are derived from laser scanning microscopy but the pink blood vessel was created and added to provide a spatial context. In figure 7b the G-protein alpha-subunit, adenyl cyclase and phosphodiesterase molecules were all extracted as 3D models from the protein data bank. The purple stream of cAMP molecules has been created and is an artistic impression. Similarly, the triglycerides inside the lipid blob of the adipocyte (figure 7c) have also been created, as was the lipid blob itself. However, both of these characters in the animation are situated inside a cell that was collected using laser scanning microscopy. The combination of art an science is extremely powerful and holds enormous potential. However, it must be handled with care as the capacity to reinforce misconceptions in the as a .nfti format using image (Figure 8). Whilst the current version does not allow extensive interaction with individual objects (within a volume) it does provide an outstanding view of a 3channel data set. Controls for brightness, contrast and image depth are also provided. At the time of writing the newest version now incorporates a collaborative option where multiple users at remote sites can examine the same dataset. This is a must-have application for anyone working with CLSM and VR.

Co-localisation studies, in mouse embryonic fibroblasts, have also been performed in VR using a fully immersive HMD. Although still in its early stages of development in user interaction, this type of work holds great promise (Theart et al., 2016).

A striking feature of examining CLSM data by walking through it and around it is the dramatic change in perspective. A data set that the user may be completely familiar with through examination on a flat screen takes on a truly different dimension when projected at a relative size of about 1.5 meters square in an interactive space. Involving the cerebellum in the learning process by physically moving around the data (for example as in Figure 8) may confer significant cognitive advantages (Buckner, 2013)

The use of game engine software for the analysis of

microscope-based data may not be obvious at first. What is a game? One definition may be; a series of task oriented movements that leads to an overall objective. The objective could be moving from A to $B$ whilst collecting objects and/or information that will assist in the next game level. A game needs a scene or environment to provide the context and the player needs to be able to interact with the scene in order to complete a task. Game design software provides pre-fabricated tools to enable quick creation of a game.

Returning to wireframe meshes and surfaces: microscope-based 3D assets can be considered as game objects or characters in a game. One standard file format for 3D game objects is .obj and this file format can be derived using the methods described previously. Figure 9 shows a scene from a game that is currently under development by the author. Each of the surfaces shown in figure 6 have been imported into UNITY Game Engine. The game also incorporates a series of pre-fabricated tools that enable player movement, teleportation, interaction and visualisation in either VR or on a flat screen. Yellow platforms and blue teleportation pads provide a means of moving around the scene in VR whilst physically occupying a fairly restricted space (i.e. the constraints of a tethered VR-HMD). It should be noted that very little (if any) coding/scripting

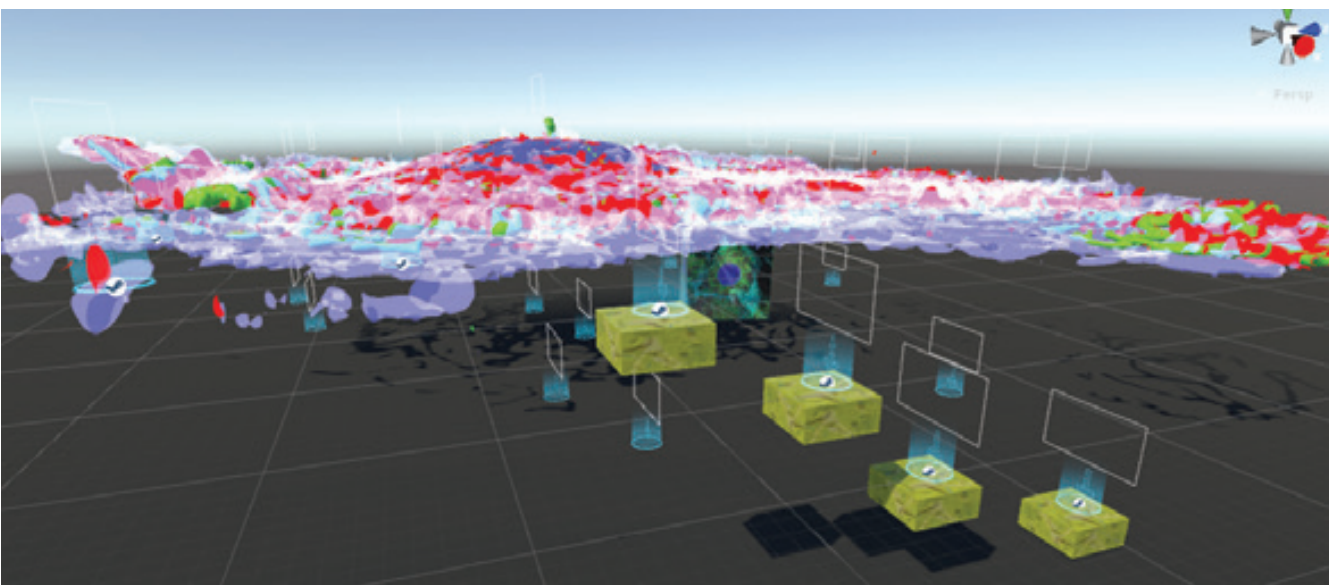

Figure 9. VR scene and/or 3D game using cellular structural data. The COS cell surfaces shown in figure $6 \mathrm{~b}$ and $6 \mathrm{c}$ have been imported into UNITY 3D. The player can explore the scene using a fully immersive VR headset. The player can 'teleport', using a hand-held controller/pointer to any of the blue circular teleport pads. The yellow blocks enable the player to climb up onto the cell and walk across its surface. The scene can
then be developed into a game by adding interactive objects and tasks. The game can be delivered as $3 D$ platform style game using a flat screen or as a fullvR game. 
is required to build a basic game. It only requires assembling a series of software development toolkits (SDKs), prefabs and 3D assets.

Gamification in education is a growing field and 'serious games' are already well established although more research is still required to identify the educational effectiveness of the various game styles and components (Mayer 2019). There exists a huge potential to exploit the masses of data that undoubtedly exist on the hard drives and within the archives of most CLSM users. Data sets that may not have been pretty enough to make it to publication could perhaps serve as modifiable 3D assets for game development. With the inevitable increase in remote learning, and research funders' demands for greater public engagement and impact game creation of scientific findings could be an attractive solution

\section{Educational Considerations}

If considerable time is to be spent making $3 \mathrm{D}$ animations, VR scenes and educational games, it is essential that these are effective learning tools. Some guidance is available in the form of cognitive load theory (CLT, Pass et al., 2003) and multimedia theory (MMT, Mayer 2014). Briefly, CLT recognises three types of cognitive load; intrinsic, extraneous and germane. At its simplest level these three loads can be interpreted as object (or in this case 'scene') complexity, design and background knowledge respectively. This is a gross simplification but will suffice for this brief discussion as it gives us broad aspects to consider. CLSM-based data will have an inherent complexity of multiple structures and features (intrinsic load). The viewer of any educational content will have a particular level of prior knowledge that can be drawn upon by working memory to create new learning (germane load). The instructional design may incorporate additiona material that places the information and tasks in context. That additional content can place an extraneous load on the learner if it is unnecessary. The three cognitive loads are additive and can lead to overload which will reduce learning. In general, for CLSM-related animations we can control all of the cognitive loading but reducing extraneous load may be the most effective when presenting a learner with a complex 3D scene.

Multimedia theory describes the use of pictures and sounds and the way in which these are integrated into the learning process. The basic idea is that (written) words and pictures are visual and so occupy the same mental processing channel. Thus it could be expected that an educational multimedia design would favour pictures and narration rather than pictures with on-screen text. Having two things to look at simultaneously would split attention. In a study conducted by the author looking at 3D-animation design preferences of a group of (36) undergraduate students the majority preferred to view an animation that had both on-screen text and narration (unpublished observations). This contravenes the 'redundancy principle' of multimedia theory and could be expected to confer an unnecessary (extraneous) cognitive load. A full discussion is outwith the scope of this article. However, suffice to say that current theories of learning and cognitive load may require to be revised to align with modern educational animation and VR content. Having a huge archive of $3 D$ assets from laser scanning microscopy and access to complex software for constructing fabulous content is therefore not enough. It is essential that serious thought is given to the instructive design of this type of content.

\section{Conclusion}

This article aims to stimulate microscopy users to think about how they might utilise their archived bank of images (2D or 3D). Although the workflow described above focuses mainly on $3 D$ there is a huge potential in creating sophisticated multimedia incorporating 2D images and using software such as Adobe After Effects or equivalent. As lecture capture and online learning grow in popularity there will come a time when lecturers (and others) will need to spend more time on content creation rather than content delivery.

This article concentrates on taking CLSM data sets through to VR visualisation but does not mention Augmented Reality (AR). At present VR is a mature product at the plateau of its technology life-cycle curve where hardware refinement and content creation is driving the market. $A R$ is well behind the curve but can be expected to catch up. Quite how $A R$ and microscopy will integrate is a matter of some speculation but a lot of what we learn on the VR journey should be applicable.

Overall, this is an exciting time for 3D (and 4D) microscopy. After a long lag, the computing power and visualisation technology can match the output of the microscopes. We are not yet at the stage of providing realtime VR visualisation of a live cell mounted on a scanning microscope, but that is surely only a matter of time.

\section{Acknowledgements}

The author wishes to acknowledge the large number of undergraduate and postgraduate students who have contributed to the development of this work over several years. The author is particularly grateful to $\mathrm{Mr}$ Paul McLeod (3D artist and animator, figure 7), Prof. Minhua Ma (collaboration on animation work) \& Prof. JC McGrath (collaboration on vascular \& confocal work)

\section{References}

Arribas, S, Daly, C.J., Gordon, J.F. \& McGrath, J.C. Confocal Myography: Applications for the study of resistance arteries. In Resistance arteries Structure and Function. Humana Press . p259-264 (1994)

Buckner R (2013) The cerebellum and cognitive function: 25 years of insight from anatomy and neuroimaging. Neutron 80, 807-815.

Daly CJ, Clunie L \& Ma M (20I4) From Microscope to Movies; 3D animations for teaching physiology. Microscopy and Analysis, 28 (6) September; 7-10.

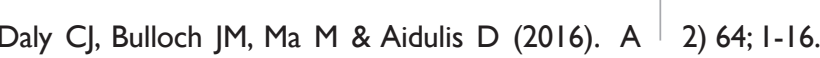

comparison of animated versus static images in an instructional multimedia presentation. Adv. Physiol. Edu. 40, 20I-205

Daly CJ (2018). The future of education? Using 3D animation and virtual reality in teaching physiology. Physiology News (PNII I), Summer Issue, p43

Draelos M, Keller B, Viehland C, Carrasco-Zevallos OM, Kuo A \& Izatt J (2018). Real-time visualization and interaction with static and live optical coherence tomography volumes in immersive virtual reality Biomedical Optics Express Vol. 9, Issue 6, pp. 2825 2843

Luo D , Barker J, J. C. McGrath \& C.J. Daly. (1998) Iterative Multilevel Thresholding and Splitting for 3D Segmentation of Live Cell Nuclei Using Laser Scanning Confocal Microscopy. J. Comp. Assisted. Microscopy.Vol. I0, Number 4 pp. I5I-162

Maolood IY, Abdulridha Al-Salhi HE, and Lu S (2018) Thresholding for Medical Image Segmentation for Cancer using Fuzzy Entropy with Level SetAlgorithm. Open Med (Wars) 13: 374-383.

Mayer RE (2014) Cognitive Theory of multimedia. New York. Cambridge University press

Mayer RE (2019). Computer games in education Annu. Rev. Psychol. 70; 22.1-22.19

Pass F, Renkl A \& Sweller J (2003). Cognitive load theory and instructional design: Recent developments. Educational Psychologist, 38(I), I-4

Pool R (2016) An animated life. Microscopy \& Analysis, editorial, Sept

Romaszewski M, Gawron P \& Opozfa S (2013) Research Dimensionality Reduction of Dynamic Mesh Animations Using HO-SVD. Journal of Artificial Intelligence and Soft Computing. Vol 3, No. 3 p277-289.

Theart RP, Loos B \& Niesler TR (2016). Virtual reality assisted microscopy data visualization and colocalization analysis. BMC Bioinformatics, 18 (suppl 